\title{
Announcement: Changes to the Table of Contents of Physical Review Accelerators and Beams
}

The editors of Physical Review Accelerators and Beams are pleased to announce the following changes to the journal's table of contents. In response to increasing interest and demand as well as to cover topics at the boundaries between disciplines, we have created new sections on "Design Studies," "Particle and Radiation Detectors," "Targets, Collimators, and Beam Dumps," "Accelerator Materials and Surfaces," "Material-Beam Interaction," and "Computing and Algorithms."

Published 18 December 2017

DOI: 10.1103/PhysRevAccelBeams.20.120001 